\title{
Hopf Bifurcation of a Mathematical Model for Growth of Tumors with an Action of Inhibitor and Two Time Delays
}

\author{
Bao Shi, ${ }^{1}$ Fangwei Zhang, ${ }^{1}$ and Shihe $X u^{2}$ \\ ${ }^{1}$ Institute of Systems Science and Mathematics, Naval Aeronautical and Astronautical University, \\ Yantai, Shandong 264001, China \\ ${ }^{2}$ Department of Mathematics, Zhaoqing University, Zhaoqing, Guangdong 526061, China
}

Correspondence should be addressed to Shihe Xu, shihexu03@yahoo.com.cn

Received 3 November 2010; Accepted 25 January 2011

Academic Editor: Allan C. Peterson

Copyright (C) 2011 Bao Shi et al. This is an open access article distributed under the Creative Commons Attribution License, which permits unrestricted use, distribution, and reproduction in any medium, provided the original work is properly cited.

\begin{abstract}
A mathematical model for growth of tumors with two discrete delays is studied. The delays, respectively, represent the time taken for cells to undergo mitosis and the time taken for the cell to modify the rate of cell loss due to apoptosis and kill of cells by the inhibitor. We show the influence of time delays on the Hopf bifurcation when one of delays is used as a bifurcation parameter.
\end{abstract}

\section{Introduction}

Within last four decades, an increasing number of partial differential equation models for tumor growth or therapy have been developed; compare [1-9] and references cited therein. Most of those models are in form of free boundary problems. Rigorous mathematical analysis of such free boundary problems has drawn great interest, and many interesting results have been established; compare [10-20] and references cited therein. Analysis of such free boundary problems not only provides a sound theoretical basis for tumor medicine, but also greatly enriches the understanding of deferential equations.

In this paper, we study a mathematical model for growth of tumors with two discrete delays. The delays, respectively, represent the time taken for cells to undergo mitosis and the time taken for the cell to modify the rate of cell loss due to apoptosis and kill of cells by the inhibitor. The model is as follows:

$$
\Delta_{r} \sigma=\Gamma_{1} \sigma, \quad 0<r<R(t), \quad t>0,
$$




$$
\begin{gathered}
\frac{\partial \sigma}{\partial r}(0, t)=0, \quad \sigma(R(t), t)=\sigma_{\infty}, \quad t>0, \\
\Delta_{r} \beta=\Gamma_{2} \beta, \quad 0<r<R(t), \quad t>0, \\
\frac{\partial \beta}{\partial r}(0, t)=0, \quad \beta(R(t), t)=\beta_{\infty}, \quad t>0, \\
\frac{d}{d t} \frac{4 \pi R^{3}(t)}{3}=4 \pi \int_{0}^{R\left(t-\tau_{1}\right)} \lambda \sigma\left(r, t-\tau_{1}\right) r^{2} d r-4 \pi \int_{0}^{R\left(t-\tau_{2}\right)}\left[\lambda \widetilde{\sigma}+\mu \beta\left(r, t-\tau_{2}\right)\right] r^{2} d r, \quad t>0, \\
R(t)=\varphi(t), \quad-\tau \leq t \leq 0,
\end{gathered}
$$

where $\Gamma_{1}, \Gamma_{2}, \lambda, \mu, \sigma_{\infty}, \beta_{\infty}, \widetilde{\sigma}, \tau_{1}$, and $\tau_{2}$ are positive constants, $\varphi$ is a given positive function. $\Delta_{r}=\left(1 / r^{2}\right)(\partial / \partial r)\left(r^{2}(\partial . / \partial r)\right)$. The term $\Gamma_{1} \sigma$ in (1.1) is the consumption rate of nutrient in a unit volume; $\Gamma_{2} \beta$ in (1.3) is the consumption rate of inhibitor in a unit volume; $\sigma_{\infty}$ reflects constant supply of nutrient that the tumor receives from its surface; $\beta_{\infty}$ reflects constant supply of inhibitor that the tumor receives from its surface. $\tau_{1}$ represents the time taken for cells to undergo mitosis, and $\tau_{2}$ represents the time taken for the cell to modify the rate of cell loss due to apoptosis and kill of cells by the inhibitor. The two terms on the right hand side of (1.5) are explained as follows: the first term is the total volume increase in unit time interval induced by cell proliferation; $\lambda \sigma$ is the cell proliferation rate in unit volume. The second term is total volume shrinkage in unit time interval caused by cell apoptosis (cell death due to aging) and the kills of cells by the inhibitor; the cell apoptosis rate is assumed to be constant;

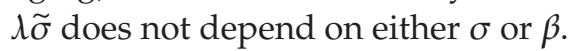

The study of effects of time delay in growth of tumors by using the method of mathematical models was initiated by Byrne [1]. Recently this study has drawn attention of some other researchers; compare Bodnar and Forys [10], Fory and Bodnar [15], Forys and Kolev [16], Sarkar and Banerjee [7] with one delay; and compare Piotrowska [18], Xu [19] with two delays. This mathematical model is established by modifying the model of Byrne [2] by considering two independent time delays effect as in $[18,19]$. The modifications are based on biological considerations, see Cui and $\mathrm{Xu}$ [14] for details. In [14], the authors studied the problem (1.1)-(1.6) with only one delay in proliferation, that is, $\tau_{2}=0$, and showed that the dynamical behavior of solutions of the model with a delay in proliferation is similar to that of solutions for corresponding nondelayed problem. The aim of this paper is to investigate the influence of time delays on the Hopf bifurcation when $\tau_{2}$ is used as a bifurcation parameter.

Denote $\theta=\sqrt{\left(\Gamma_{2} / \Gamma_{1}\right)}$. By rescaling the space variable, we may assume that $\Gamma_{1}=1$. Accordingly, we have $\theta=\sqrt{\Gamma_{2}}$. The solution of (1.1)-(1.4) is

$$
\sigma(r, t)=\frac{\sigma_{\infty} R(t)}{\sinh R(t)} \frac{\sinh r}{r}, \quad \beta(r, t)=\frac{\beta_{\infty} R(t)}{\sinh (\theta R(t))} \frac{\sinh (\theta r)}{r}
$$

Substituting (1.7) into (1.6) we obtain

$$
\dot{R}(t)=R(t)\left[\lambda \sigma_{\infty} p\left(R\left(t-\tau_{1}\right)\right)\left(\frac{R\left(t-\tau_{1}\right)}{R(t)}\right)^{3}-\left(\mu \beta_{\infty} p\left(R\left(t-\tau_{2}\right)\right)+\frac{1}{3} \lambda \widetilde{\sigma}\right)\left(\frac{R\left(t-\tau_{2}\right)}{R(t)}\right)^{3}\right]
$$


here $p(x)=(x \operatorname{coth} x-1) / x^{2}$. Set $\omega(t)=R^{3}(t)$, and we have

$$
\dot{\omega}(t)=3 \lambda \sigma_{\infty} p\left(\omega^{1 / 3}\left(t-\tau_{1}\right)\right) \omega\left(t-\tau_{1}\right)-\left(3 \mu \beta_{\infty} p\left(\omega^{1 / 3}\left(t-\tau_{2}\right)\right)+\lambda \widetilde{\sigma}\right) \omega\left(t-\tau_{2}\right) .
$$

Using the step method (see, e.g., [21]), we can easily show that if there exists a solution for $t \in\left[(n-1) \tau_{3}, n \tau_{3}\right]$, then the solution for $t \in\left[n \tau_{3},(n+1) \tau_{3}\right]$, where $n \in N, \tau_{3}=\min \left(\tau_{1}, \tau_{2}\right)$, is defined by the formula

$\omega(t)=\omega\left(n \tau_{3}\right)+\int_{n \tau_{3}}^{t} 3 \lambda \sigma_{\infty} p\left(\omega^{1 / 3}\left(s-\tau_{1}\right)\right) \omega\left(s-\tau_{1}\right)-\left(3 \mu \beta_{\infty} p\left(\omega^{1 / 3}\left(s-\tau_{2}\right)\right)+\lambda \tilde{\sigma}\right) \omega\left(s-\tau_{2}\right) d s$.

Clearly the step method gives the existence of unique solution to (1.9) because of $s-\tau_{1}, s-\tau_{2} \in$ $\left[(n-1) \tau_{3}, n \tau_{3}\right]$.

Using Theorem 1.2 from [22], we can get nonnegative initial condition $\omega^{0}$, and the solution of (1.9) can become negative in a finite time. Therefore, through the rest of the paper we assume that a positive solution of (1.9) with initial function $\omega^{0}$ exists for every $t>0$.

\section{Stability of the Stationary Solutions and Existence of Local Hopf Bifurcation}

In this section, we will study stability of the stationary solutions and existence of local Hopf bifurcation.

The first step is to find stationary solutions. Stationary solutions to (1.9) satisfy the equation

$$
\left(3 \lambda \sigma_{\infty} p\left(x^{1 / 3}\right)-3 \mu \beta_{\infty} p\left(\theta x^{1 / 3}\right)-\lambda \tilde{\sigma}\right) x=0
$$

Clearly, (2.1) has the trivial solution $x=0$. Next, we consider the positive solutions to (2.1). From [17] we know that $p(x)$ is strictly monotone decreasing for $x>0$, and

$$
\lim _{x \rightarrow 0+} p(x)=\frac{1}{3}, \quad \lim _{x \rightarrow \infty} p(x)=0 .
$$

Let $g(x)=3 \lambda \sigma_{\infty} p\left(x^{1 / 3}\right)-3 \mu \beta_{\infty} p\left(\theta x^{1 / 3}\right)-\lambda \widetilde{\sigma}, x>0$. Then

$$
\begin{gathered}
\lim _{x \rightarrow 0+} g(x)=\lambda \sigma_{\infty}-\mu \beta_{\infty}-\lambda \tilde{\sigma}, \\
g^{\prime}(x)=x^{-2 / 3}\left(\lambda \sigma_{\infty} p^{\prime}\left(x^{1 / 3}\right)-\theta \mu \beta_{\infty} p^{\prime}\left(\theta x^{1 / 3}\right)\right)=-\theta \mu \beta_{\infty} x^{-2 / 3} p^{\prime}\left(x^{1 / 3}\right)\left(\frac{p^{\prime}\left(\theta x^{1 / 3}\right)}{p^{\prime}\left(x^{1 / 3}\right)}-\frac{\lambda \sigma_{\infty}}{\theta \mu \beta_{\infty}}\right) .
\end{gathered}
$$


By [12], we know that $p^{\prime}(\theta y) / p^{\prime}(y)$ is strictly monotone increasing (resp., decreasing) if $0<$ $\theta<1$ (resp., $\theta>1$ ) and

$$
\lim _{y \rightarrow 0+} \frac{p^{\prime}(\theta y)}{p^{\prime}(y)}=\theta, \quad \lim _{y \rightarrow \infty} \frac{p^{\prime}(\theta y)}{p^{\prime}(y)}=\frac{1}{\theta^{2}}
$$

Using these results, we can easily prove the following lemma (see [11] or [14]).

Lemma 2.1. Assume that $0<\theta<1$. Then the following assertions hold.

(1) If $\beta_{\infty} \geq \lambda \sigma_{\infty} / \theta^{2} \mu$ then there exist no positive solutions for (2.1), that is, the problem (1.9) has no positive stationary solutions.

(2) If $\beta_{\infty}<\lambda \sigma_{\infty} / \theta^{2} \mu$, then in the case $\widetilde{\sigma} \geq \sigma_{\infty}-\mu \beta_{\infty} / \lambda$ there exist no positive solutions for (2.1), that is, the problem (1.9) has no positive stationary solutions, and in the opposite case $\tilde{\sigma}<\sigma_{\infty}-\mu \beta_{\infty} / \lambda$, there exists a unique positive solution $x=\omega_{s}$ for (2.1), that is, the problem (1.9) has a unique positive stationary solution $x=\omega_{s}$. Moreover $g^{\prime}\left(\omega_{s}\right)<0$.

Assume that $\theta>1$. Then the following assertions hold.

(3) If $\beta_{\infty} \geq \theta \lambda \sigma_{\infty} / \mu$ then there exist no positive solutions for (2.1), that is, the problem (1.9) has no positive stationary solutions.

(4) If $\beta_{\infty} \leq \lambda \sigma_{\infty} / \theta^{2} \mu$, then in the case $\widetilde{\sigma} \geq \sigma_{\infty}-\mu \beta_{\infty} / \lambda$ there exist no positive solutions for (2.1), that is, the problem (1.9) has no positive stationary solution, and in the opposite case $\tilde{\sigma}<\sigma_{\infty}-\mu \beta_{\infty} / \lambda$, there exists a unique positive solution $x=\omega_{s}$ for (2.1), that is, the problem (1.9) has a unique positive stationary solution. Moreover $g^{\prime}\left(\omega_{s}\right)<0$.

(5) If $\lambda \sigma_{\infty} / \theta^{2} \mu<\beta_{\infty}<\theta \lambda \sigma_{\infty} / \mu$, then there exists a unique $x^{*}>0$ such that

$$
\frac{p^{\prime}\left(\theta y^{*}\right)}{p^{\prime}\left(y^{*}\right)}=\frac{\lambda \sigma_{\infty}}{\theta \mu \beta_{\infty}}
$$

here $y^{*}=\left(x^{*}\right)^{1 / 3}$, and $x^{*}$ is the maximum point of $g(x)$. Denote $g\left(x^{*}\right)=M$. Then if $\widetilde{\sigma}>3 M$ there exist no positive solutions for (2.1), that is, the problem (1.9) has no positive stationary solutions. If $0<\widetilde{\sigma} \leq \sigma_{\infty}-\mu \beta_{\infty} / \lambda$ there exists a unique positive solution $x=\omega_{s}$ for (2.1) which satisfy $g^{\prime}\left(\omega_{s}\right)<0$, that is, the problem (1.9) has a unique positive stationary solution satisfying $g^{\prime}\left(\omega_{s}\right)<0$. If $\sigma_{\infty}-\mu \beta_{\infty} / \lambda<\tilde{\sigma}<3 M$ there exist two positive solutions $x_{1}=\omega_{s 1}<x_{1}=\omega_{s 2}$ for (2.1) which satisfy $g^{\prime}\left(\omega_{s 1}\right)>0, g^{\prime}\left(\omega_{s 2}\right)<0$, that is, the problem (1.9) has two positive stationary solutions satisfying $g^{\prime}\left(\omega_{s 1}\right)>0, g^{\prime}\left(\omega_{s 2}\right)<0$, respectively.

The next step is to study the stability and the Hopf bifurcation of (1.9). Linearizing (1.9) at positive stationary solutions, we obtain

$$
\dot{\omega}(t)=-A_{1} \omega\left(t-\tau_{1}\right)-A_{2} \omega\left(t-\tau_{2}\right)
$$

where $A_{1}=-\lambda \sigma_{\infty}\left[\omega_{s}^{1 / 3} p^{\prime}\left(\omega_{s}^{1 / 3}\right)+3 p\left(\omega_{s}^{1 / 3}\right)\right], A_{2}=\mu \beta_{\infty}\left(\theta \omega_{s}^{1 / 3} p^{\prime}\left(\theta \omega_{s}^{1 / 3}\right)+3 p\left(\theta \omega_{s}^{1 / 3}\right)\right)+\lambda \widetilde{\sigma}$ 
Similarly linearizing (1.9) at the trivial stationary solution we get

$$
\dot{\omega}(t)=-B_{1} \omega\left(t-\tau_{1}\right)-B_{2} \omega\left(t-\tau_{2}\right)
$$

where $B_{1}=-\lambda \sigma_{\infty}, B_{2}=\mu \beta_{\infty}+\lambda \widetilde{\sigma}$.

We claim $A_{1}<0, A_{2}>0$. Actually, for $y>0, y^{3} p(y)$ is strictly monotone increasing in $y$ (see [14]), that is, for $y>0$,

$$
\frac{d\left(y^{3} p(y)\right)}{d y}>0 \Longleftrightarrow y^{2}(y \dot{p}(y)+3 p(y))>0 \Longleftrightarrow y \dot{p}(y)+3 p(y)>0
$$

This readily implies that $A_{1}=-\left.\lambda \sigma_{\infty}(y \dot{p}(y)+3 p(y))\right|_{y=\omega_{s}^{1 / 3}}<0$. Immediately, $A_{2}=\mu \beta_{\infty}(y \dot{p}(y)+$ $3 p(y))\left.\right|_{y=\theta \omega_{s}^{1 / 3}}+\widetilde{\sigma}>0$. Hence the claim is true.

The characteristic of (2.6) is as follows:

$$
z=-A_{1} e^{-z \tau_{1}}-A_{2} e^{-z \tau_{2}}
$$

From [14] we know that if $\tau_{2}=0$ then for arbitrary $\tau_{1}>0$, the dynamical behavior of solutions of problem (1.9) with nonnegative initial function is similar to that of solutions for corresponding nondelayed problem. By continuity, for sufficiently small $\tau_{2}>0$, the dynamical behavior of solutions of problem (1.9) with nonnegative initial function is also similar to that of solutions for corresponding nonretarded problem.

In the following, we will study stability of the stationary solutions and existence of local Hopf bifurcation. From biological point of view it is reasonable to take $\tau_{2}$ as bifurcation parameter, for detail see [18] and the references therein.

The case when $B_{1}<0, B_{2}>0$ was studied in $[23,24]$, and the proof of the following lemma can be found in it.

Lemma 2.2. Consider the equation

$$
\dot{x}(t)=f\left(x\left(t-\tau_{1}\right), x\left(t-\tau_{2}\right)\right),
$$

with a nonnegative initial continuous function $\varphi:[-\tau, 0] \rightarrow R_{+}$, where $\tau_{1}, \tau_{2}$ are the positive constants, $\tau=\max \left(\tau_{1}, \tau_{2}\right)$, and $f$ is a continuously differentiable nonlinear function. Assume that (2.10) has the trivial stationary solution, that is, $f(0,0)=0$. Let the linearized equation around the trivial solution of (2.10) be as follows:

$$
\dot{x}(t)=-B_{1} x\left(t-\tau_{1}\right)-B_{2} x\left(t-\tau_{2}\right) .
$$

Then

(1) if $B_{1}<0, B_{2}>\left|B_{1}\right|$, and $\tau_{1} \in\left(0, \pi / 2 \sqrt{B_{2}^{2}-B_{1}^{2}}\right]$, then there exists $\tau_{2}^{0}>0$ such that for $\tau_{2} \in\left[0, \tau_{2}^{0}\right)$ the trivial solution to (2.10) is asymptotically stable and for $\tau_{2}=\tau_{2}^{0}$ the Hopf bifurcation occurs;

(2) if $B_{1}<0,0<B_{2}<\left|B_{1}\right|$, the trivial solution to (2.10) is unstable independently on the values of both delays, and there is no Hopf bifurcation. 
Use Lemma 2.2, we easily have the following.

Corollary 2.3. Consider the equation

$$
\dot{x}(t)=f\left(x\left(t-\tau_{1}\right), x\left(t-\tau_{2}\right)\right),
$$

with a nonnegative initial continuous function $\varphi:[-\tau, 0] \rightarrow R_{+}$, where $\tau_{1}, \tau_{2}$ are the positive constants, $\tau=\max \left(\tau_{1}, \tau_{2}\right), f$ is a continuously differentiable nonlinear function. Assume that (2.12) has the positive stationary solution $x=x_{s}$, that is, $f\left(x_{s}, x_{s}\right)=0$. Let the linearized equation around the positive stationary solution of (2.10) be as follows:

$$
\dot{x}(t)=-A_{1} x\left(t-\tau_{1}\right)-A_{2} x\left(t-\tau_{2}\right) .
$$

Then

(1) if $A_{1}<0, A_{2}>\left|A_{1}\right|$, and $\tau_{1} \in\left(0, \pi / 2 \sqrt{A_{2}^{2}-A_{1}^{2}}\right]$, then there exists $\tau_{2}^{0}>0$ such that for $\tau_{2} \in\left[0, \tau_{2}^{0}\right)$ the positive stationary solution to $(2.10)$ is asymptotically stable and for $\tau_{2}=\tau_{2}^{0}$ the Hopf bifurcation occurs;

(2) if $A_{1}<0,0<A_{2}<\left|A_{1}\right|$, the positive stationary solution to (2.10) is unstable independently on the values of both delays, and there is no Hopf bifurcation.

Noticing $A_{1}=-\lambda \sigma_{\infty}\left[\omega_{s}^{1 / 3} p^{\prime}\left(\omega_{s}^{1 / 3}\right)+3 p\left(\omega_{s}^{1 / 3}\right)\right], A_{2}=\mu \beta_{\infty}\left(\theta \omega_{s}^{1 / 3} p^{\prime}\left(\theta \omega_{s}^{1 / 3}\right)+3 p\left(\theta \omega_{s}^{1 / 3}\right)\right)+$ $\tilde{\sigma}$ and $\omega_{s}>0$ satisfy $(2.1)$, by direct computation, we have

$$
A_{2}-\left|A_{1}\right|=A_{2}+A_{1}=-\lambda \sigma_{\infty} p^{\prime}\left(\omega_{s}^{1 / 3}\right)+\theta \mu \beta_{\infty} p^{\prime}\left(\theta \omega_{s}^{1 / 3}\right) .
$$

Since $p(x)$ is strictly monotone decreasing for $x>0$, we are readily get

$$
\begin{aligned}
& A_{2}>\left|A_{1}\right| \Longleftrightarrow \frac{p^{\prime}\left(\theta \omega_{s}^{1 / 3}\right)}{p^{\prime}\left(\omega_{s}^{1 / 3}\right)}<\frac{\lambda \sigma_{\infty}}{\theta \mu \beta_{\infty}} \Longleftrightarrow g^{\prime}\left(\omega_{s}\right)<0, \\
& A_{2}<\left|A_{1}\right| \Longleftrightarrow \frac{p^{\prime}\left(\theta \omega_{s}^{1 / 3}\right)}{p^{\prime}\left(\omega_{s}^{1 / 3}\right)}>\frac{\lambda \sigma_{\infty}}{\theta \mu \beta_{\infty}} \Longleftrightarrow g^{\prime}\left(\omega_{s}\right)>0 .
\end{aligned}
$$

Clearly $B_{1}<0$, by direct computation, we obtain

$$
\begin{aligned}
& B_{2}>\left|B_{1}\right| \Longleftrightarrow \widetilde{\sigma}>\sigma_{\infty}-\frac{\mu \beta_{\infty}}{\lambda}, \\
& B_{2}<\left|B_{1}\right| \Longleftrightarrow \tilde{\sigma}<\sigma_{\infty}-\frac{\mu \beta_{\infty}}{\lambda} .
\end{aligned}
$$


Noticing

$$
\begin{aligned}
& \beta_{\infty}<\frac{\lambda}{\mu}\left(\sigma_{\infty}-\tilde{\sigma}\right) \Longleftrightarrow \tilde{\sigma}>\sigma_{\infty}-\frac{\mu \beta_{\infty}}{\lambda} \Longleftrightarrow B_{2}>\left|B_{1}\right|, \\
& \beta_{\infty}>\frac{\lambda}{\mu}\left(\sigma_{\infty}-\tilde{\sigma}\right) \Longleftrightarrow \tilde{\sigma}<\sigma_{\infty}-\frac{\mu \beta_{\infty}}{\lambda} \Longleftrightarrow B_{2}<\left|B_{1}\right|,
\end{aligned}
$$

by Lemma 2.2, we can conclude the following.

(i) Assume that $\beta_{\infty}<(\lambda / \mu)\left(\sigma_{\infty}-\widetilde{\sigma}\right)$ and $\tau_{1} \in\left(0, \pi / 2 \sqrt{B_{2}^{2}-B_{1}^{2}}\right]$ hold, then there exists $\tau_{2}^{0}>0$ such that for $\tau_{2} \in\left[0, \tau_{2}^{0}\right)$ the trivial solution to (1.9) is asymptotically stable and for $\tau_{2}=\tau_{2}^{0}$ the Hopf bifurcation occurs.

(ii) Assume that $\beta_{\infty}>(\lambda / \mu)\left(\sigma_{\infty}-\tilde{\sigma}\right)$ holds, the trivial solution to (1.9) is unstable independently on the values of both delays, and there is no Hopf bifurcation.

By simple computation, we have

$$
\begin{aligned}
& \beta_{\infty}<\frac{\lambda}{\mu}\left(\sigma_{\infty}-\tilde{\sigma}\right), \quad \beta_{\infty}<\frac{\lambda \sigma_{\infty}}{\mu \theta^{2}} \Longleftrightarrow \\
& 0<\theta<1, \quad \beta_{\infty}<\frac{\lambda \sigma_{\infty}}{\mu \theta^{2}}, \quad \tilde{\sigma}<\sigma_{\infty}-\frac{\mu \beta_{\infty}}{\lambda} \quad \text { or } \quad \theta>1, \quad \beta_{\infty}<\frac{\lambda \sigma_{\infty}}{\mu \theta^{2}}, \quad \tilde{\sigma}<\sigma_{\infty}-\frac{\mu \beta_{\infty}}{\lambda}
\end{aligned}
$$

$\Rightarrow(1.9)$ has a positive stationary solution $\omega_{s}$ and $g^{\prime}\left(\omega_{s}\right)<0 \Leftrightarrow A_{2}>\left|A_{1}\right|$. Then by Corollary 2.3, we have the following. Assume that $\beta_{\infty}<(\lambda / \mu)\left(\sigma_{\infty}-\tilde{\sigma}\right)$ and $\beta_{\infty}<\lambda \sigma_{\infty} / \mu \theta^{2}$ hold, then for $\tau_{1} \in\left(0, \pi / 2 \sqrt{A_{2}^{2}-A_{1}^{2}}\right]$ there exists $\tau_{2}^{0}>0$ such that for $\tau_{2} \in\left[0, \tau_{2}^{0}\right)$ the positive stationary solution to (1.9) is asymptotically stable and for $\tau_{2}=\tau_{2}^{0}$ the Hopf bifurcation occurs.

Since $0<\theta<1$ and $(\lambda / \mu)\left(\sigma_{\infty}-\tilde{\sigma}\right)<\beta_{\infty}<\lambda \sigma_{\infty} / \mu \theta^{2} \Leftrightarrow \beta_{\infty}<\lambda \sigma_{\infty} / \mu \theta^{2}, \tilde{\sigma}>\sigma_{\infty}-$ $\mu \beta_{\infty} / \lambda \Rightarrow B_{2}>\left|B_{1}\right|$. From Lemma 2.1 we know that if $0<\theta<1$ and $(\lambda / \mu)\left(\sigma_{\infty}-\tilde{\sigma}\right)<\beta_{\infty}<$ $\lambda \sigma_{\infty} / \mu \theta^{2}$ hold, then (1.9) has no positive stationary solution. By Lemma 2.2, we readily have the following. Assume that $0<\theta<1$ and $(\lambda / \mu)\left(\sigma_{\infty}-\tilde{\sigma}\right)<\beta_{\infty}<\lambda \sigma_{\infty} / \mu \theta^{2}$ hold, then for $\tau_{1} \in\left(0, \pi / 2 \sqrt{B_{2}^{2}-B_{1}^{2}}\right]$ there exists $\tau_{2}^{0}>0$ such that for $\tau_{2} \in\left[0, \tau_{2}^{0}\right)$ the trivial solution to (2.10) is asymptotically stable and for $\tau_{2}=\tau_{2}^{0}$ the Hopf bifurcation occurs.

Assume that $\theta>1$, then we have the following.

If $\beta_{\infty}>\left(\theta \lambda \sigma_{\infty} / \mu\right)\left(>\lambda \sigma_{\infty} / \mu \theta^{2}\right) \Rightarrow \beta_{\infty}<(\lambda / \mu)\left(\sigma_{\infty}-\tilde{\sigma}\right) \Rightarrow B_{2}>\left|B_{1}\right| ;$

if $(\lambda / \mu)\left(\sigma_{\infty}-\tilde{\sigma}\right)<\beta_{\infty}<\lambda \sigma_{\infty} / \mu \theta^{2} \Rightarrow \tilde{\sigma}>\sigma_{\infty}-\mu \beta_{\infty} / \lambda \Leftrightarrow B_{2}>\left|B_{1}\right|$;

if $\lambda \sigma_{\infty} / \mu \theta^{2}<\beta_{\infty}<\theta \lambda \sigma_{\infty} / \mu$ and if $\tilde{\sigma}>3 M \Rightarrow \tilde{\sigma}<\sigma_{\infty}-\mu \beta_{\infty} / \lambda \Leftrightarrow B_{2}<\left|B_{1}\right|$; if $\tilde{\sigma}<\sigma_{\infty}-\mu \beta_{\infty} / \lambda \Rightarrow B_{2}>\left|B_{1}\right|$; if $\sigma_{\infty}-\mu \beta_{\infty} / \lambda<\tilde{\sigma}<3 M \Rightarrow g^{\prime}\left(\omega_{s 2}\right)<0$ and then by Lemma 2.2 and Corollary 2.3, we have the following.

(1) Assume that $\theta>1, \beta_{\infty}>\theta \lambda \sigma_{\infty} / \mu$ or $\theta>1,(\lambda / \mu)\left(\sigma_{\infty}-\tilde{\sigma}\right)<\beta_{\infty}<\lambda \sigma_{\infty} / \mu \theta^{2}$ holds, then for $\tau_{1} \in\left(0, \pi / 2 \sqrt{B_{2}^{2}-B_{1}^{2}}\right]$ there exists $\tau_{2}^{0}>0$ such that for $\tau_{2} \in\left[0, \tau_{2}^{0}\right)$ the trivial solution to (2.10) is asymptotically stable and for $\tau_{2}=\tau_{2}^{0}$ the Hopf bifurcation occurs. 
(2) Assume that $\theta>1,(\lambda / \mu)\left(\sigma_{\infty}-\tilde{\sigma}\right)<\beta_{\infty}<\lambda \sigma_{\infty} / \mu \theta^{2}$ hold. Then if $\tilde{\sigma}>3 M$, the trivial solution to (1.9) is unstable independently on the values of both delays, and there is no Hopf bifurcation; if $\tilde{\sigma}<\sigma_{\infty}-\mu \beta_{\infty} / \lambda$, then for $\tau_{1} \in\left(0, \pi / 2 \sqrt{B_{2}^{2}-B_{1}^{2}}\right.$ ] there exists $\tau_{2}^{0}>0$ such that for $\tau_{2} \in\left[0, \tau_{2}^{0}\right)$ the trivial solution to (2.10) is asymptotically stable and for $\tau_{2}=\tau_{2}^{0}$ the Hopf bifurcation occurs; if $\sigma_{\infty}-\mu \beta_{\infty} / \lambda<\widetilde{\sigma}<3 M$, then for $\tau_{1} \in\left(0, \pi / 2 \sqrt{A_{2}^{2}-A_{1}^{2}}\right]$ there exists $\tau_{2}^{0}>0$ such that for $\tau_{2} \in\left[0, \tau_{2}^{0}\right)$ the positive stationary solution $\omega_{s 2}$ to (1.9) is asymptotically stable and for $\tau_{2}=\tau_{2}^{0}$ the Hopf bifurcation occurs.

We summarize as follows.

Theorem 2.4. (i) Assume that $\beta_{\infty}<(\lambda / \mu)\left(\sigma_{\infty}-\tilde{\sigma}\right)$ and $\tau_{1} \in\left(0, \pi / 2 \sqrt{B_{2}^{2}-B_{1}^{2}}\right]$ hold, then there exists $\tau_{2}^{0}>0$ such that for $\tau_{2} \in\left[0, \tau_{2}^{0}\right)$ the trivial solution to (1.9) is asymptotically stable and for $\tau_{2}=\tau_{2}^{0}$ the Hopf bifurcation occurs.

(ii) Assume that $\beta_{\infty}>(\lambda / \mu)\left(\sigma_{\infty}-\tilde{\sigma}\right)$ holds, the trivial solution to (1.9) is unstable independently on the values of both delays, and there is no Hopf bifurcation.

(iii) Assume that $\beta_{\infty}<(\lambda / \mu)\left(\sigma_{\infty}-\tilde{\sigma}\right)$ and $\beta_{\infty}<\lambda \sigma_{\infty} / \mu \theta^{2}$ hold, then for $\tau_{1} \in$ $\left(0, \pi / 2 \sqrt{A_{2}^{2}-A_{1}^{2}}\right]$ there exists $\tau_{2}^{0}>0$ such that for $\tau_{2} \in\left[0, \tau_{2}^{0}\right)$ the positive stationary solution to (1.9) is asymptotically stable and for $\tau_{2}=\tau_{2}^{0}$ the Hopf bifurcation occurs.

(iv) Assume that $0<\theta<1$ and $(\lambda / \mu)\left(\sigma_{\infty}-\widetilde{\sigma}\right)<\beta_{\infty}<\lambda \sigma_{\infty} / \mu \theta^{2}$ hold, then for $\tau_{1} \in$ $\left(0, \pi / 2 \sqrt{B_{2}^{2}-B_{1}^{2}}\right]$ there exists $\tau_{2}^{0}>0$ such that for $\tau_{2} \in\left[0, \tau_{2}^{0}\right)$ the trivial solution to $(2.10)$ is asymptotically stable and for $\tau_{2}=\tau_{2}^{0}$ the Hopf bifurcation occurs.

(v) Assume that $\theta>1, \beta_{\infty}>\theta \lambda \sigma_{\infty} / \mu$ or $\theta>1,(\lambda / \mu)\left(\sigma_{\infty}-\tilde{\sigma}\right)<\beta_{\infty}<\lambda \sigma_{\infty} / \mu \theta^{2}$ holds, then for $\tau_{1} \in\left(0, \pi / 2 \sqrt{B_{2}^{2}-B_{1}^{2}}\right]$ there exists $\tau_{2}^{0}>0$ such that for $\tau_{2} \in\left[0, \tau_{2}^{0}\right)$ the trivial solution to (2.10) is asymptotically stable and for $\tau_{2}=\tau_{2}^{0}$ the Hopf bifurcation occurs.

(vi) Assume that $\theta>1, \lambda \sigma_{\infty} / \mu \theta^{2}<\beta_{\infty}<\theta \lambda \sigma_{\infty} / \mu$ hold. Then if $\tilde{\sigma}>3 M$, the trivial solution to (1.9) is unstable independently on the values of both delays, and there is no Hopf bifurcation; if $\tilde{\sigma}<\sigma_{\infty}-\mu \beta_{\infty} / \lambda$, then for $\tau_{1} \in\left(0, \pi / 2 \sqrt{B_{2}^{2}-B_{1}^{2}}\right]$ there exists $\tau_{2}^{0}>0$ such that for $\tau_{2} \in\left[0, \tau_{2}^{0}\right)$ the trivial solution to (2.10) is asymptotically stable and for $\tau_{2}=\tau_{2}^{0}$ the Hopf bifurcation occurs; if $\sigma_{\infty}-\mu \beta_{\infty} / \lambda<\tilde{\sigma}<3 M$, then for $\tau_{1} \in\left(0, \pi / 2 \sqrt{A_{2}^{2}-A_{1}^{2}}\right]$ there exists $\tau_{2}^{0}>0$ such that for $\tau_{2} \in\left[0, \tau_{2}^{0}\right)$ the positive stationary solution $\omega_{s 2}$ to $(1.9)$ is asymptotically stable and for $\tau_{2}=\tau_{2}^{0}$ the Hopf bifurcation occurs.

\section{Conclusion}

In this paper, we study a mathematical model for growth of tumors with two discrete delays. The delays, respectively, represent the time taken for cells to undergo mitosis and the time taken for the cell to modify the rate of cell loss due to apoptosis and kill of cells by the inhibitor. Final mathematical formulation of the model is retarded differential equation of the form

$$
\dot{\omega}(t)=f\left(\omega\left(t-\tau_{1}\right), \omega\left(t-\tau_{2}\right)\right),
$$


with a nonnegative initial continuous function $\varphi:[-\tau, 0] \rightarrow R_{+}$, where $\tau_{1}, \tau_{2}$, and $\tau=$ $\max \left(\tau_{1}, \tau_{2}\right)$ are the positive constants, $f$ is a continuously differentiable function. The results show that the two independent delays control the dynamics of the solution of the problem (3.1) and the dynamic behavior is different to the corresponding nonretarded ordinary equation

$$
\dot{\omega}(t)=f(\omega(t), \omega(t)), \quad x(0)=x_{0}>0,
$$

or retarded differential equation with only one delay of the form

$$
\dot{\omega}(t)=f(\omega(t-\tau), \omega(t)),
$$

with a nonnegative initial continuous function $\varphi:[-\tau, 0] \rightarrow R_{+}$. However the dynamic behaviors of the problem (3.2) and (3.3) are similar, see [14].

\section{Acknowledgments}

The work of the third author is partially supported by NNSF (10926128), NSF, and YMF of Guangdong Province (9251064101000015, LYM10133).

\section{References}

[1] H. M. Byrne, "The effect of time delays on the dynamics of avascular tumor growth," Mathematical Biosciences, vol. 144, no. 2, pp. 83-117, 1997.

[2] H. M. Byrne, "Growth of nonnecrotic tumors in the presence and absence of inhibitors," Mathematical Biosciences, vol. 130, no. 2, pp. 151-181, 1995.

[3] H. M. Byrne and M. A. J. Chaplain, "Growth of necrotic tumors in the presence and absence of inhibitors," Mathematical Biosciences, vol. 135, no. 2, pp. 187-216, 1996.

[4] M. J. Dorie, R. F. Kallman, D. F. Rapacchietta et al., "Migration and internalization of cells and polystyrene microspheres in tumor cell spheroids," Experimental Cell Research, vol. 141, no. 1, pp. 201209, 1982.

[5] H. Greenspan, "Models for the growth of solid tumor by diffusion," Studies in Applied Mathematics, vol. 51, pp. 317-340, 1972.

[6] H. P. Greenspan, "On the growth and stability of cell cultures and solid tumors," Journal of Theoretical Biology, vol. 56, no. 1, pp. 229-242, 1976.

[7] R. R. Sarkar and S. Banerjee, "A time delay model for control of malignant tumor growth," in National Conference on Nonlinear Systems and Dynamics, pp. 1-4, 2006.

[8] K. Thompson and H. Byrne, "Modelling the internalisation of labelled cells in tumor spheroids," Bulletin of Mathematical Biology, vol. 61, pp. 601-623, 1999.

[9] J. Ward and J. King, "Mathematical modelling of avascular-tumor growth II: modelling grwoth saturation," IMA Journal of Mathematics Applied in Medicine and Biology, vol. 15, pp. 1-42, 1998.

[10] M. Bodnar and U. Forys, "Time delay in necrotic core formation," Mathematical Biosciences and Engineering, vol. 2, no. 3, pp. 461-472, 2005.

[11] S. Cui, "Analysis of a mathematical model for the growth of tumors under the action of external inhibitors," Journal of Mathematical Biology, vol. 44, no. 5, pp. 395-426, 2002.

[12] S. Cui and A. Friedman, "Analysis of a mathematical model of the effect of inhibitors on the growth of tumors," Mathematical Biosciences, vol. 164, no. 2, pp. 103-137, 2000.

[13] S. Cui and A. Friedman, "Analysis of a mathematical model of the growth of necrotic tumors," Journal of Mathematical Analysis and Applications, vol. 255, no. 2, pp. 636-677, 2001.

[14] S. Cui and S. Xu, "Analysis of mathematical models for the growth of tumors with time delays in cell proliferation," Journal of Mathematical Analysis and Applications, vol. 336, no. 1, pp. 523-541, 2007. 
[15] U. Fory and M. Bodnar, "Time delays in proliferation process for solid avascular tumour," Mathematical and Computer Modelling, vol. 37, no. 11, pp. 1201-1209, 2003.

[16] U. Foryś and M. Kolev, "Time delays in proliferation and apoptosis for solid avascular tumour," in Mathematical Modelling of Population Dynamics, vol. 63 of Banach Center Publications, pp. 187-196, Polish Academy of Sciences, Warsaw, Poland, 2004.

[17] A. Friedman and F. Reitich, "Analysis of a mathematical model for the growth of tumors," Journal of Mathematical Biology, vol. 38, no. 3, pp. 262-284, 1999.

[18] M. J. Piotrowska, "Hopf bifurcation in a solid avascular tumour growth model with two discrete delays," Mathematical and Computer Modelling, vol. 47, no. 5-6, pp. 597-603, 2008.

[19] S. Xu, "Hopf bifurcation of a free boundary problem modeling tumor growth with two time delays," Chaos, Solitons \& Fractals, vol. 41, no. 5, pp. 2491-2494, 2009.

[20] S. Xu, "Analysis of tumor growth under direct effect of inhibitors with time delays in proliferation," Nonlinear Analysis: Real World Applications B, vol. 11, no. 1, pp. 401-406, 2010.

[21] J. Hale, Theory of Functional Differential Equations, Springer, New York, NY, USA, 2nd edition, 1977, Applied Mathematical Sciences.

[22] M. Bodnar, "The nonnegativity of solutions of delay differential equations," Applied Mathematics Letters, vol. 13, no. 6, pp. 91-95, 2000.

[23] X. Li, S. Ruan, and J. Wei, "Stability and bifurcation in delay-differential equations with two delays," Journal of Mathematical Analysis and Applications, vol. 236, no. 2, pp. 254-280, 1999.

[24] M. J. Piotrowska, "A remark on the ODE with two discrete delays," Journal of Mathematical Analysis and Applications, vol. 329, no. 1, pp. 664-676, 2007. 


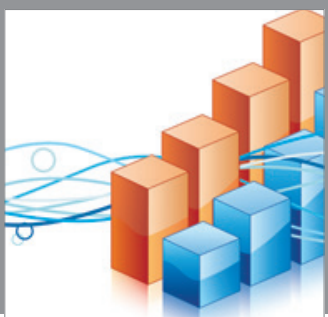

Advances in

Operations Research

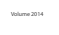

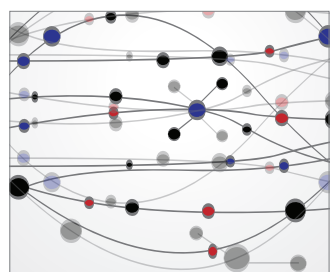

\section{The Scientific} World Journal
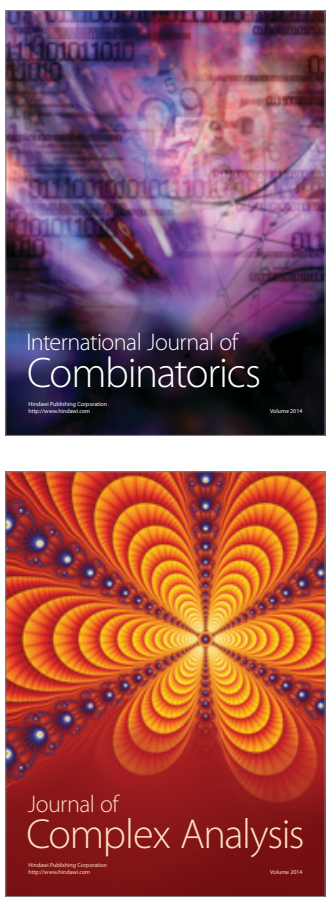

International Journal of

Mathematics and

Mathematical

Sciences
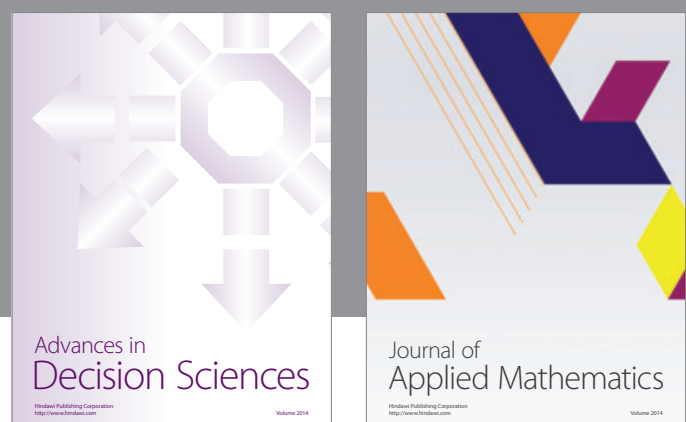

Journal of

Applied Mathematics
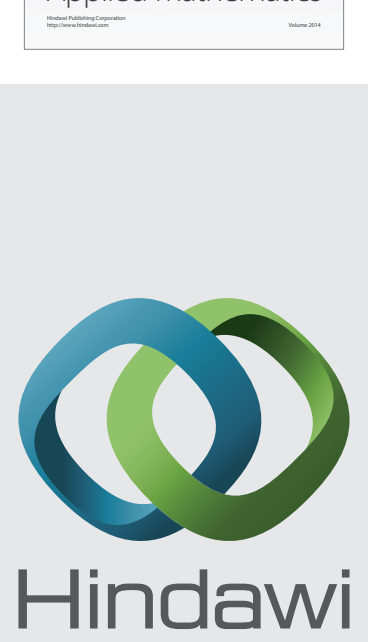

Submit your manuscripts at http://www.hindawi.com
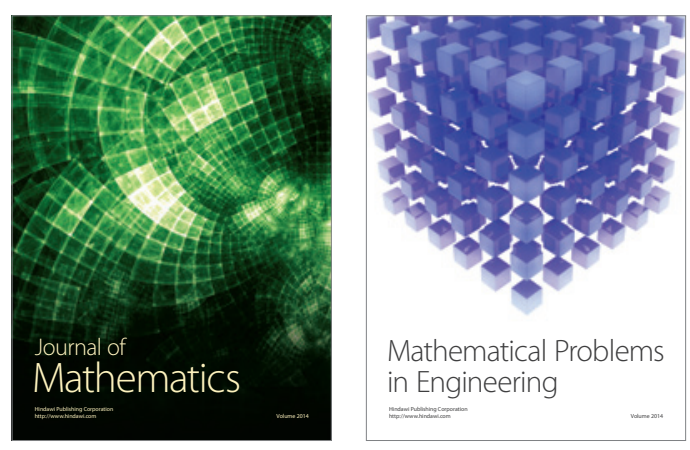

Mathematical Problems in Engineering
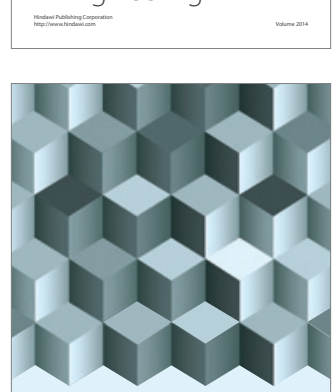

Journal of

Function Spaces
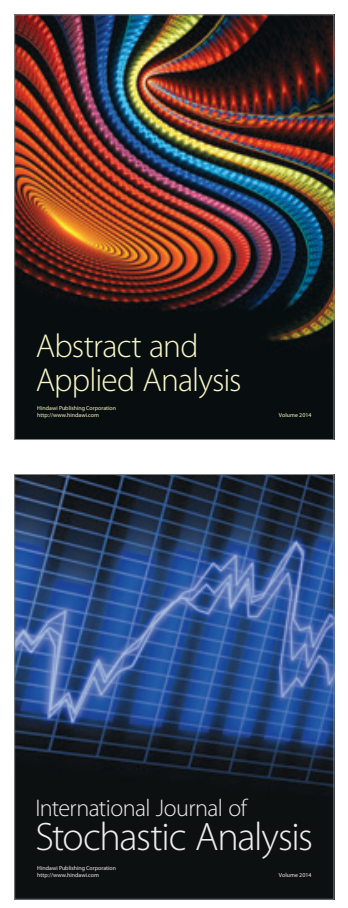

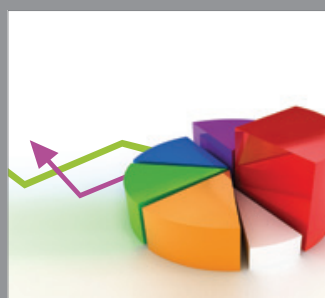

ournal of

Probability and Statistics

Promensencen
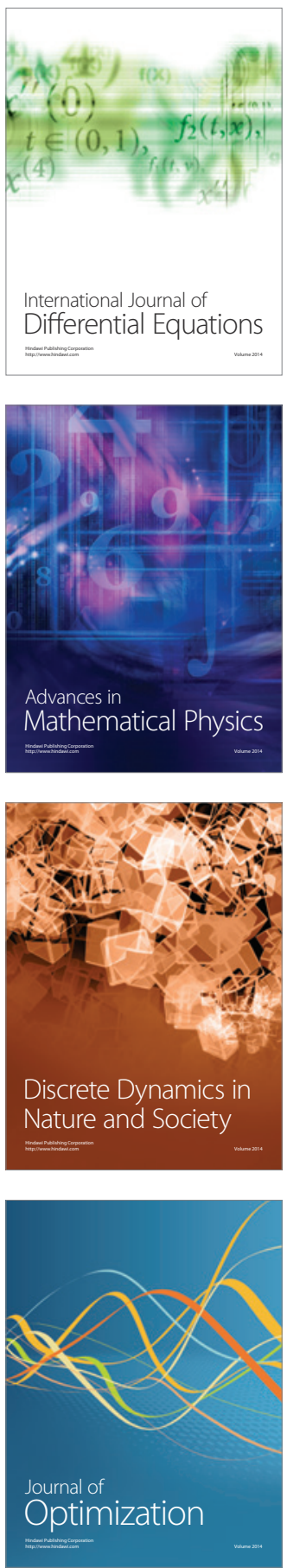